\title{
Diseño e implementación de tareas de alta demanda cognitiva basadas en la sucesión look and say
}

\author{
Víctor Manero, Universidad de Zaragoza (España) \\ José M. Muñoz-Escolano, Universidad de Zaragoza (España) \\ Antonio M. Oller-Marcén, Centro Universitario de la Defensa de Zaragoza (España)
}

\section{Diseño e implementación de tareas de alta demanda cognitiva basadas en la sucesión look and say}

\section{Resumen}

Pese a que su tratamiento escolar usual se centra en aspectos principalmente de cálculo, las sucesiones son un tópico matemático con el potencial para desarrollar en los alumnos aspectos del razonamiento matemático. En este trabajo se diseña una secuencia de tareas de alta demanda cognitiva basadas en la sucesión 'look and say' y se implementa en un grupo de secundaria con especial interés por las matemáticas durante una sesión del Taller de Talento Matemático en la Universidad de Zaragoza. La metodología es exploratoria y descriptiva con análisis mixto de datos cualitativos. Los alumnos participantes resolvieron las tareas con un alto grado de éxito y surgieron bastantes respuestas de gran riqueza conceptual. Estas tareas pueden ser útiles para trabajar aspectos transversales del currículo e identificar alumnos con altas capacidades matemáticas.

Palabras clave. Sucesiones; 'look and say'; diseño de tareas; alta demanda cognitiva; tipos de razonamiento.

Design and implementation of cognitive high-demand tasks based on the look and say sequence

\section{Abstract}

Even though their school treatment is mainly based on calculations, numerical sequences are a mathematical topic with the potential to develop aspects of mathematical reasoning amongst students. In this work, we design a sequence of tasks of cognitive high-demand based on the 'look and say' sequence and implement them with a group of secondary school students particularly interested in mathematics during a session of the Workshop of Mathematical Talent at the University of Zaragoza. The methodology is exploratory and descriptive with mixed analysis of qualitative data. Participants solved the tasks with a high rate of success and several answers were of high conceptual richness. These tasks might be useful to work transversal curricular aspects and to identify those mathematically gifted.

Keywords. Sequences; 'look and say'; task design; cognitive high-demand; types of reasoning.

\section{Introducción}

La resolución de problemas en los que aparecen sucesiones numéricas es un contenido matemático clásico. Ya en el Papiro de Rhind encontramos un problema cuya resolución consiste en la suma de los cinco primeros términos de una progresión geométrica de razón 7 (Chace, 1986, p. 112). Prácticamente en todos los textos sobre aritmética, en cualquier cultura y momento histórico, es posible hallar problemas que involucran progresiones aritméticas o geométricas. Recordemos también que la sucesión de Fibonacci aparece en el Liber Abaci de 1202 (Sigler, 2002, pp. 404-405).

Esta tradición se ha mantenido y el trabajo con sucesiones es un clásico de la matemática escolar. En España aparecen en el currículo de 3. ${ }^{\circ}$ de Educación Secundaria Obligatoria (14-15 años), que especifica los siguientes contenidos: "Sucesiones numéricas. Sucesiones recurrentes. Progresiones aritméticas y geométricas" (BOE, 3 de enero de 2015, p. 392). En paralelo, se enumeran estos estándares de aprendizaje: 
- Calcula términos de una sucesión numérica recurrente usando la ley de formación a partir de términos anteriores.

- Obtiene una ley de formación o fórmula para el término general de una sucesión sencilla de números enteros o fraccionarios.

- Identifica progresiones aritméticas y geométricas, expresa su término general, calcula la suma de los " $n$ " primeros términos, y las emplea para resolver problemas.

- Valora e identifica la presencia recurrente de las sucesiones en la naturaleza y resuelve problemas asociados a las mismas.

Aparentemente, en los libros de texto se suele poner el énfasis en las progresiones aritméticas y geométricas. Según Bajo Benito et al. (2015) esto lleva a muchos alumnos a identificar erróneamente el concepto de sucesión con el de progresión. Por otra parte, se suele dejar de lado el trabajo de contenidos como las sucesiones recurrentes, que podrían ser un ámbito adecuado para trabajar aspectos interesantes que aparecen recogidos en el Bloque I del currículo (búsqueda y reconocimiento de patrones, razonamiento inductivo y deductivo, uso de TIC, etc.) y que suponen una entrada a los procesos de iteración y recursión, áreas fundamentales de la matemática discreta (Weigand, 2004). Biza et al. (2020) señalan que la investigación sobre las tareas de generalización de patrones mediante sucesiones cuyo término general es una expresión algebraica lineal está muy desarrollada en la literatura científica, puesto que son tareas frecuentes en la introducción del álgebra escolar, y apuntan la necesidad de continuar investigando con tareas donde los términos de la sucesión no provengan de sucesiones lineales.

Desde un punto de vista cognitivo, Przenioslo (2006) distingue una doble naturaleza en cuanto a la comprensión de los estudiantes del concepto sucesión: por un lado, como listado de números $\mathrm{y}$, por otro, como función cuyo dominio de definición se encuentra en los números naturales. Ambas facetas son importantes ya que la noción de límite de sucesiones está estrechamente vinculada a la concepción de sucesión como función y de serie numérica (Arnal-Palacián, 2019; Delgado et al., 2015) y es fundamental para articular una correcta comprensión del concepto de límite en matemáticas, junto con el límite funcional en el infinito y el límite funcional en un punto (Fernández-Plaza y Simpson, 2016). Bajo Benito et al. (2019) estudian la comprensión de estudiantes de la sucesión como listado de números y caracterizan niveles, en los cuales las sucesiones recurrentes son identificadas como elementos matemáticos que aparecen en respuestas de los estudiantes que poseen niveles avanzados de comprensión y las incluyen dentro de una descomposición genética del concepto de sucesión numérica.

En su trabajo sobre sucesiones recurrentes y modos de representación, Weigand (2004) adapta a las sucesiones el modelo de Vollrath con cinco niveles de comprensión: intuitiva (conocer ejemplos y representaciones de sucesiones), orientada al contenido (conocer propiedades de sucesiones como la monotonía y la convergencia), integrada (relacionar propiedades de las sucesiones con conceptos como funciones o aplicaciones), formal (conocer definiciones y ser capaz de probar propiedades de las sucesiones) y estructural (trabajar con composiciones de sucesiones o apreciarlas como elementos de un espacio vectorial).

Biza et al. (2020) estudian estrategias que emplean profesores en formación al encontrar el término general de sucesiones cuadráticas mediante eye-tracking, si las sucesiones se dan en forma de tablas, pares ordenados o una sucesión de datos con comas. 
Los resultados señalan que, en todas las representaciones de la sucesión, la estrategia más común que en un principio emplean dichos profesores es la de estudiar la diferencia entre un término y el anterior en la variable dependiente.

Con el presente estudio exploramos la posibilidad de trabajar con sucesiones numéricas recurrentes que se definen de forma sencilla, pero no son habituales en clase. Los objetivos son de diseño, implementación y análisis: i) diseñar una secuencia de tareas con alta demanda cognitiva basada en el trabajo con sucesiones numéricas fácilmente definibles, no habituales en clase, que fomente distintos tipos de razonamiento; ii) implementar la secuencia con un grupo de estudiantes de Educación Secundaria especialmente interesados en las matemáticas; y iii) analizar los resultados.

Las tareas diseñadas podrían fomentar la aparición en respuestas de los participantes de rasgos propios de alumnos con altas capacidades matemáticas, por lo que podrían servir como instrumento para identificar estos alumnos.

\section{Sucesiones look and say y su versión ordenada}

El matemático británico J. H. Conway (1986) popularizo la sucesión look and say. Los primeros términos de esta sucesión son: 1, 11, 21, 1211, 111221, 312211...

A partir de un valor inicial (en este caso el 1), cada término consiste en la descripción verbal del término anterior. Así, dado el valor inicial 35, podemos describirlo verbalmente como "un tres y un cinco", por lo que el siguiente término de la sucesión es 1315. Este término se puede describir verbalmente como "un uno, un tres, un uno y un cinco"; dando lugar al siguiente término 11131115. Aplicando esta regla se obtiene la sucesión look and say de valor inicial 35.

Las sucesiones anteriores admiten una sencilla modificación que da lugar a las llamadas sucesiones look and say ordenadas. La modificación consiste en que, en cada paso, no se describe verbalmente el término anterior, sino que se cuenta primero el número total de unos, luego el total de doses, luego el número de treses y así sucesivamente. Por ejemplo, partiendo del valor inicial 35, el segundo término vuelve a ser 1315, pero entonces el siguiente es 211315 (“dos unos, un tres y un cinco"), el siguiente es 31121315 ("tres unos, un dos, un tres y un cinco"), etc.

Bronstein y Fraenkel (1994) y Saurberg y Shu (1997) han estudiado estas sucesiones. Entre otras propiedades, son eventualmente periódicas; es decir, después de un número finito de términos, toda sucesión look and say ordenada presenta ciclos periódicos de longitud finita. Ahora, dado un término cualquiera, siempre es posible encontrar predecesores, que llamaremos "triviales", utilizando la idea de que el término $b b b b \ldots b b b b$ formado por $n$ repeticiones de la cifra $b$, es un término anterior a cualquier término $n b$. Por ejemplo, dado el término 111213, las expresiones verbales de algunos predecesores triviales serían "ciento once doses y un tres" y "once mil ciento veintiún treses". Para evitar este tipo de situaciones, los autores citados utilizan distintos sistemas de representación, generalmente vinculados al uso de $n$-tuplas.

Dejando los casos triviales, en la sucesión look and say no ordenada, cada término tiene a lo sumo un predecesor, mientras que el predecesor puede no ser único en la sucesión ordenada. Por ejemplo, el término anterior a 111213 en la sucesión no ordenada es 123; en la ordenada es cualquier permutación de las cifras de 123.

En cuanto a su utilización con fines didácticos, estas sucesiones son fáciles de plantear y no requieren de conocimientos específicos sobre sucesiones. Además, no son progresiones de tipo aritmético, ni geométrico, ni existe una expresión algebraica 
de su término general. Barton et al. (2004) describen las buenas propiedades que poseen este tipo de sucesiones para que estudiantes universitarios desarrollen sus primeras experiencias de investigación, al estudiar propiedades como su monotonía y convergencia al variar el primer término de la sucesión.

Para definir y desarrollar estas sucesiones se emplean de manera espontánea dos modos de representación: la escrita y oral de los términos. Por el contrario, por el rápido crecimiento de los términos, no es sencillo que aparezcan representaciones gráficas y razonamientos visuales, como los apuntados por Alcock y Simpson (2004), que caracterizan a estudiantes que tratan los constructos matemáticos como objetos, establecen conclusiones rápidas y están convencidos de que éstas son correctas.

En Gairín et al. (2018) se describe una tarea basada en estas sucesiones cuya implementación con alumnos de Bachillerato proporcionó información para la mejora del diseño de la secuencia y para focalizar el análisis de respuestas de los estudiantes.

\section{Descomposición genética del concepto de sucesión en la Teoría APOE}

Dentro del modelo APOE (Dubinsky y McDonald, 2001), Asiala et al. (1996) definen la descomposición genética de un objeto como "un conjunto estructurado de constructos mentales que podría describir el modo en que un concepto se desarrolla en la mente de un individuo" (p. 7). Estos autores indican que la obtención de la descomposición genética de un objeto es fruto de un análisis teórico orientado a desarrollar un modelo cognitivo del objeto y que debe ser testada experimentalmente.

Las descomposiciones genéticas juegan un papel clave en la investigación basada en el modelo APOE, proporcionando "las bases para el diseño de instrumentos para obtener y analizar datos de los estudiantes" (Arnon et al., 2014, p. 37). Este constructo también puede jugar un papel importante en el diseño instruccional (Jojo, 2014). Así, Trigueros y Oktaç (2019) señalan la importancia de que el diseño de tareas contemple los elementos matemáticos incluidos en la descomposición genética de los conceptos matemáticos para promover mayor comprensión en el alumnado.

En el contexto de las sucesiones, Bajo Benito et al. (2019, pp. 153-154) construyen una descomposición genética del concepto de sucesión entendida como lista numérica. Esta descomposición genética consta de seis elementos ordenados jerárquicamente:

1. Prerrequisitos (expresión algebraica, valor numérico, representación de puntos en la recta numérica y el plano cartesiano)

2. Cálculo de un término a partir de su posición

3. Interiorización de la acción anterior como proceso

4. Inversión del proceso anterior para obtener la posición de un elemento

5. Coordinación del proceso del punto 2 en los modos de representación

6. Encapsulación del proceso anterior como objeto, de modo que se puedan estudiar propiedades globales (para todos los términos de la sucesión)

7. Desencapsulación del objeto anterior en un proceso que permita considerar la sucesión completa y términos concretos

\section{Tipos de razonamiento}

Peirce (1934) distingue y caracteriza tres tipos de razonamiento: deductivo, inductivo y abductivo. Aunque la posición del autor con respecto a estos tipos varió a lo largo de su obra, en sus Lectures on Pragmatism (Peirce, 1934, p. 171), los describe así: "Deduction proves that something must be; induction shows that something actually is operative; abduction merely suggests that something may be". Peirce otorgaba un 
papel especial al razonamiento abductivo al afirmar que "Abduction is the process of forming an explanatory hypothesis. It is the only logical operation which introduces any new idea" (Peirce, 1934, p. 171). En matemáticas, "proof is deductive, but the discovering and conjecturing processes is often characterized by abductive argumentation" (Pedemonte y Reid, 2011, p. 282).

Pedemonte (2007) muestra la utilidad del modelo de Toulmin (2003, pp. 89-100) para analizar la estructura de los tipos de razonamiento en Peirce, así como para describirlos esquemáticamente. En su versión simple, el modelo de Toulmin involucra datos (D), conclusiones (C) y garantías (W). Este modelo se refina introduciendo elementos como calificadores (Q), refutaciones (R) o respaldos (B) (Figura 1).

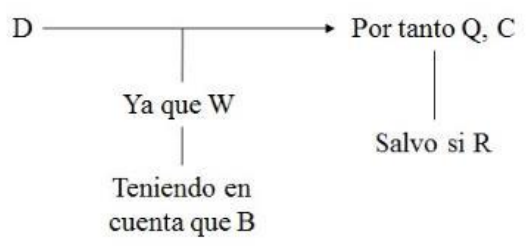

Figura 1. Modelo de Toulmin (2003, p. 97)

Adaptando el trabajo de Manrique y Soler-Álvarez (2014), la Figura 1 se puede particularizar del siguiente modo en el caso de los tipos de razonamiento en Peirce:

- Razonamiento abductivo

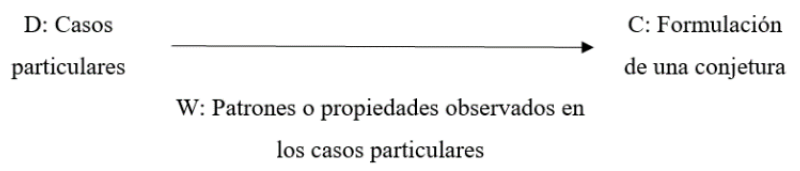

- Razonamiento inductivo

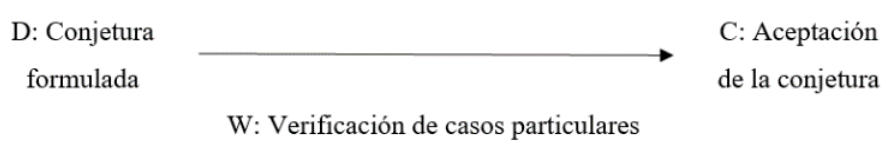

- Razonamiento deductivo

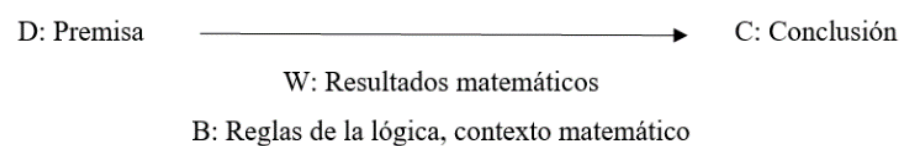

Estos diagramas ilustran una cierta secuenciación o concatenación en el proceso de investigación según el método científico. Así, la abducción propone hipótesis o conjeturas que se evalúan y ponen a prueba inductivamente y se utilizan, si son aceptadas, para explicar y realizar predicciones (Aguayo, 2011). Además de su importancia desde el punto de vista de los procesos científicos, los tipos de razonamiento son útiles en el análisis de procesos argumentativos y pueden jugar un papel en la formación de profesorado (Arce y Conejo, 2019).

Manrique y Soler-Álvarez (2014) distinguen cinco tipos de razonamiento abductivo en función de la naturaleza de los datos (gráfica o numérica), del número de casos particulares (uno o varios) o del tipo de garantía (si involucra una abducción previa o 
no). Se habla de abducción: de patrones, por analogía, con base en una característica observada en un caso particular, con diagramas dinámicos, y con base en relaciones observadas en un diagrama geométrico. Los tres primeros pueden darse en un contexto numérico y sus características son las siguientes:

- Abducción de patrones. Los datos son varios casos particulares y la garantía es el patrón (o patrones) identificado en los mismos.

- Abducción con base en una característica observada en un caso particular. Los datos son un caso particular y la garantía es una característica o propiedad observada en dicho caso.

- Abducción por analogía. Los datos son un caso particular y la garantía es una relación con un hecho conocido o con una abducción previa.

La diferencia entre los dos primeros radica en el número de casos particulares, mientras que en el tercero la diferencia estriba en el uso como garantía de lo observado en los datos y de su relación con propiedades conocidas u obtenidas previamente.

\section{Demanda cognitiva de tareas}

A la hora de establecer cuándo una tarea matemática es apropiada, Smith y Stein (1998) determinan varios factores como el nivel de los estudiantes a los que va dirigida, sus conocimientos o edad. Una tarea será apropiada si posee el potencial de llevar a los estudiantes a pensamientos de alto nivel. Con base en investigaciones previas, estos autores distinguen cuatro niveles de tareas según demanda cognitiva: de memorización, de procedimientos sin conexiones, de procedimientos con conexiones y que implican "hacer matemáticas". Los dos primeros niveles se corresponden con tareas de baja demanda cognitiva y los dos últimos con tareas de alta demanda cognitiva, siendo estas últimas las que permitan llegar a pensamientos de alto nivel. La Tabla 1 presenta indicadores de niveles de demanda cognitiva de una tarea.

Tabla 1. Algunos indicadores de niveles de demanda cognitiva (Smith y Stein, 1998, p. 348)

Nivel Indicadores

Memorización

Implican la reproducción de hechos, reglas o fórmulas ya aprendidos

No son ambiguas y lo que debe reproducirse está claramente indicado

Procedimientos

Se centran en producir respuestas correctas

sin conexiones

No tienen conexión con los conceptos subyacentes al procedimiento en uso No requieren explicación o esta se centra en describir el procedimiento

Procedimientos Se representan de diversas formas y se conectan modos de representación con conexiones Centran la atención en uso de procedimientos para desarrollar mayores niveles de comprensión de ideas y conceptos matemáticos

Requieren de un pensamiento complejo y no algorítmico

"Hacer mateRequieren exploración de conceptos, procesos y relaciones máticas" Requieren análisis de la tarea en busca de restricciones que limiten posibles soluciones y estrategias

Requieren un considerable esfuerzo cognitivo y pueden provocar ansiedad 


\section{Método}

El trabajo realizado es un estudio de caso de carácter descriptivo y exploratorio (Yin, 2018, pp. 380-381) con metodología mixta. Tomamos un diseño convergente paralelo (Creswell, 2012, p. 540) en el que se recogen y analizan datos tanto de tipo cuantitativo como cualitativo. En términos generales, la investigación se estructuró en tres fases: diseño de las tareas, implementación y recogida de información y análisis de los datos. A continuación, detallamos los aspectos metodológicos de cada fase.

\subsection{Diseño}

En el diseño y secuenciación de tareas se han considerado la descomposición genética del concepto de sucesión de Bajo Benito et al. (2019) y los niveles de demanda cognitiva de Smith y Stein (1998). Las tareas se han diseñado con potencial para involucrar razonamientos abductivos, inductivos y deductivos (Peirce, 1934).

Con respecto a la descomposición genética, en el caso de sucesiones look and say no se manejarán expresiones algebraicas. Sí podremos adoptar la descomposición anterior considerando entre los prerrequisitos la regla de formación de la sucesión. El punto 5 (sección 3) tendrá poco peso porque, aunque distintos modos de representación de los términos (oral y escrito) surgen de manera espontánea desde las primeras tareas, no tienen impacto directo en el diseño de las tareas.

Teniendo en cuenta la clasificación de tareas matemáticas de Yeo (2007, p. 19), optamos por plantear tareas investigativas y abiertas desde el punto de vista de sus objetivos y métodos de resolución. Además, las respuestas son abiertas, por lo que se ha decidido guiar a los alumnos mediante diversas tareas parciales en cada tarea.

\subsection{Implementación y recogida de información}

Las tareas diseñadas se implementaron con un grupo de 31 alumnos de $4 .^{\circ}$ curso de ESO en el Taller de Talento Matemático (de la Cueva, 2016) de la Universidad de Zaragoza. La sesión fue de 90 minutos en la tarde del 4 de mayo de 2018. Los alumnos trabajaron en equipos ( 9 de tres alumnos y 1 de cuatro). La agrupación fue aleatoria, con un número del 1 al 10 asignado a cada alumno a medida que entraba en el aula.

Durante los primeros 20 minutos se introdujo la sesión en gran grupo. Se preguntó a los alumnos por su conocimiento acerca de sucesiones y mediante unas diapositivas nombraban el término siguiente de algunas progresiones aritméticas y geométricas. Tras ello, se habló brevemente de otras sucesiones que no fueran progresiones ni aritméticas ni geométricas, mencionando la de Fibonacci. Luego se presentó la sucesión look and say, que algunos alumnos conocían, y se introdujeron las sucesiones look and say ordenadas. Tras esta introducción, que involucra el punto 1 de la descomposición genética, se realizaron las tareas en grupos. Se repartieron entre los grupos fichas en las que debían dejar constancia escrita del trabajo. Las fichas de cada grupo diferían en los valores numéricos proporcionados como datos. Durante esta segunda fase de la implementación, los grupos trabajaron de forma autónoma, si bien los dos primeros autores respondían dudas mientras supervisaban y observaban.

La principal fuente de datos fueron las producciones de los alumnos en las fichas que entregaron los grupos y en las que, además de las respuestas a las preguntas, se pedía que indicaran sus justificaciones. De forma complementaria y para puntualmente triangular análisis posteriores, los investigadores participantes en la implementación realizaron observaciones, inmediatas y diferidas (Postic y de Ketele, 1988). 


\subsection{Análisis de producciones de alumnos}

El análisis de las producciones en las respuestas a las tareas de las fichas entregadas siguió un enfoque doble. Por una parte, se llevó a cabo un análisis descriptivo del contenido de las respuestas de los alumnos. En este caso, nos centramos en aspectos de las respuestas como aciertos y errores detectados, o bien propiedades y conjeturas realizadas por los grupos. Este análisis es de carácter inductivo y no pretende (pues el tamaño de la muestra tampoco lo permitiría) generar una categorización de las respuestas. Por otro lado, cuando se solicitaban justificaciones, estas se han analizado de forma interpretativa, identificando los tipos de razonamiento presentes con el modelo de Toulmin como instrumento.

\section{Diseño de las tareas}

Teniendo en cuenta las restricciones de tiempo en la sesión de trabajo para la implementación, las tres tareas fueron las siguientes:

- Tarea 1: Formular una conjetura sobre el comportamiento asintótico de la sucesión

- Tarea 2: Comprobar la conjetura sobre el comportamiento asintótico de la sucesión y búsqueda de propiedades adicionales

- Tarea 3: Usar las propiedades para el estudio del problema inverso

\subsection{Tarea 1}

El objetivo de esta tarea es que los alumnos estudien el comportamiento a largo plazo de las sucesiones look and say ordenadas llegando a formular una conjetura sobre su carácter periódico (tras un número de términos), así como sobre la existencia de periodos de distintas longitudes. Desde el punto de vista de la descomposición genética descrita, se abordan principalmente los puntos 2 y 3 y parcialmente el 6 . Para su implementación, la tarea se subdivide en:

- Tarea 1.1. A partir de los cinco primeros términos de la sucesión con valor inicial 1, pregunta cuáles son los términos $6 .^{\circ}, 13 .^{\circ}$ y $35 .^{\circ}$ de esa sucesión.

- Tarea 1.2. Da un valor inicial (distinto del 1) que también origina un periodo de longitud 1 y pregunta por los términos $2 .^{\circ}, 13 .^{\circ}$ y $100 .^{\circ}$ de esa sucesión.

- Tarea 1.3. Da otro valor inicial que origina un periodo de longitud 2 o 3 y pregunta por los términos $2 .^{\circ}, 13 .^{\circ}$ y $100 .^{\circ}$ de esa sucesión.

Las Tareas 1.1 a 1.3 son cerradas, puesto que se proporciona un valor inicial determinado. Observamos que esta tarea implica fundamentalmente aspectos relacionados con el razonamiento abductivo (Figura 2).

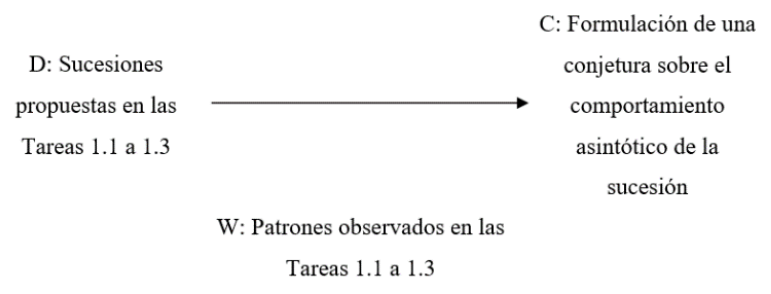

Figura 2. Modelo de Toulmin aplicado a Tarea 1 
Desde el punto de vista de la demanda cognitiva, la Tarea 1 se mueve en los niveles bajos. La primera parte de cada una de las tres tareas (cálculo de términos relativamente bajos de la sucesión) se ubica en el nivel de memorización, ya que no son ambiguas e involucran la reproducción de reglas previamente trabajadas. La segunda parte (cálculo de un término lejano) se ubica en el nivel de procedimientos sin conexiones, ya que no se establecen conexiones con conceptos subyacentes y las explicaciones que se requieren se centran en describir el procedimiento utilizado.

\subsection{Tarea 2}

Para verificar la conjetura obtenida en la tarea anterior, introducimos un applet que facilitará el cálculo de los términos de las sucesiones look and say ordenadas con distintos valores iniciales. Creamos una web con información básica, contextualización y un applet de cálculo de sucesiones look and say ordenadas. Este applet, a partir del valor inicial de la sucesión y del número de términos a calcular, devuelve por pantalla el número de términos solicitado. Para acceder a la web que aloja el applet, se proyecta un código QR (Figura 3) que los alumnos leen con su móvil.

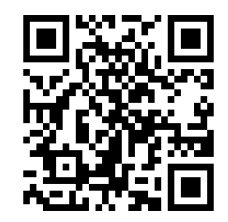

Figura 3. Código QR de la página web con la "calculadora look and say"

La Tarea 2 se lleva a cabo con la ayuda de esta herramienta. Desde el punto de vista de la descomposición genética descrita, se abordan principalmente los puntos 6 y 7 . Para su implementación se subdivide en:

- Tarea 2.1. Elegir distintos valores iniciales arbitrarios y, para cada uno, calcular los términos $13 .^{\circ}$ y $100 .^{\circ}$ de la sucesión correspondiente.

- Tarea 2.2. Conjeturar el comportamiento a largo plazo de estas sucesiones.

La Tarea 2.1 es de exploración y deja a los alumnos que experimenten. Se usa el ordenador para generar un número suficiente de casos particulares que permitan eventualmente la confirmación de la conjetura formulada en la tarea anterior. Cierra la Tarea 2.2 retomando la pregunta que constituye el objetivo. El razonamiento inductivo (Figura 4) es el que predomina.

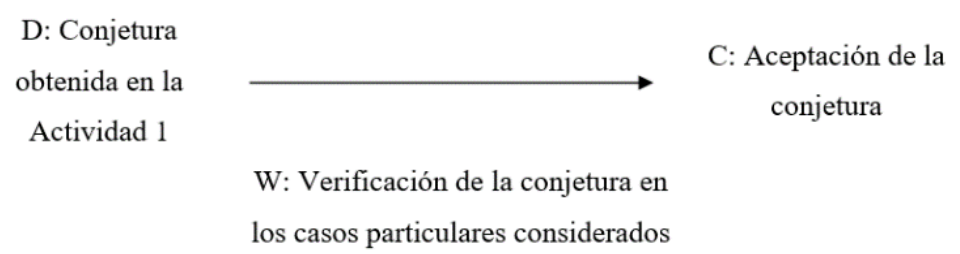

Figura 4. Modelo de Toulmin aplicado a Tarea 2

Desde el punto de vista de la demanda cognitiva, la Tarea 2 tiene dos partes. La tarea 2.1 se corresponde con un nivel de memorización y es de baja demanda cognitiva por los mismos motivos que en la tarea anterior. La tarea 2.2 es de alta demanda cognitiva y se corresponde con un nivel de procedimientos con conexiones, dado que centra 
la atención de los estudiantes en el uso de procedimientos para desarrollar mayores niveles de comprensión de ideas y conceptos matemáticos.

\subsection{Tarea 3}

Esta tarea aborda el cálculo de posibles términos anteriores a uno dado, por lo que es un problema de carácter más cerrado y abstracto. Obviando los casos triviales, el término anterior a uno dado puede no existir o no ser único. El objetivo es que los alumnos sean capaces de utilizar las propiedades descubiertas y aceptadas en las tareas anteriores. Desde el punto de vista de la descomposición genética descrita, se abordan principalmente los puntos 4, 6 y 7. Para su implementación se subdivide en:

- Tarea 3.1. Indicar propiedades o características especiales de los elementos de las sucesiones look and say.

- Tarea 3.2. Calcular el término anterior a un valor dado en tres casos: uno en el que existe y es único, otro en el que existe y no es único, y otro en el que no existe.

- Tarea 3.3. Calcular el término anterior del anterior a un valor dado en dos casos: en uno existe y en otro no.

La tarea 3.1 tiene un carácter más abierto y sirve como recopilación de propiedades descubiertas en las tareas anteriores. Las tareas 3.2 y 3.3 se basan en la aplicación de estas propiedades en la búsqueda de los términos solicitados. Esta Tarea 3 involucra principalmente razonamientos de tipo deductivo (Figura 5).

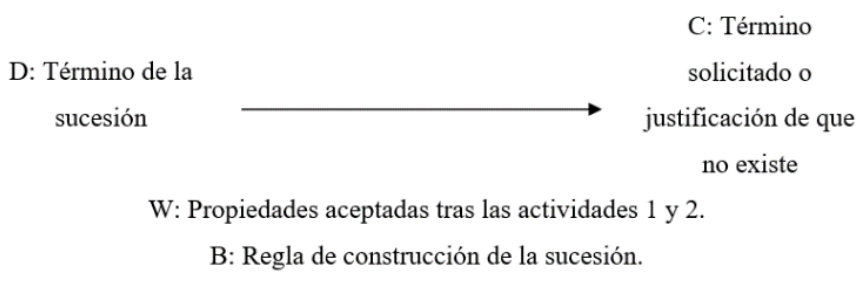

Figura 5. Modelo de Toulmin aplicado a Tarea 3

Desde el punto de vista de la demanda cognitiva, las tres subtareas de la Tarea 3 son de alta demanda cognitiva y se ubican en el nivel de "hacer matemáticas". Todas ellas requieren de un pensamiento complejo y no-algorítmico, necesitan de una cierta exploración y exigen que los estudiantes analicen la tarea buscando restricciones que limiten posibles estrategias de resolución.

\section{Resultados de la implementación}

\subsection{Resultados relativos a la Tarea 1}

Tarea 1.1. Todos los grupos calculan correctamente los términos solicitados. Hasta el $13 .^{\circ}$ término no se produce ninguna repetición, por lo que todos los grupos debieron calcular a mano esos 13 primeros términos. A partir de ese término son todos ya iguales, lo cual indicaron todos los grupos menos dos en su respuesta al cálculo del término $35 .^{\circ}$. De los 8 grupos que aportan una explicación, todos menos uno, indican que a partir del $13 .^{\circ}$ la sucesión es periódica. El grupo restante habla de "al llegar a un número de términos" de forma genérica (Figura 6). 
¿Cuál es el término $35^{\circ}$ de la sucesión? Justificad la respuesta

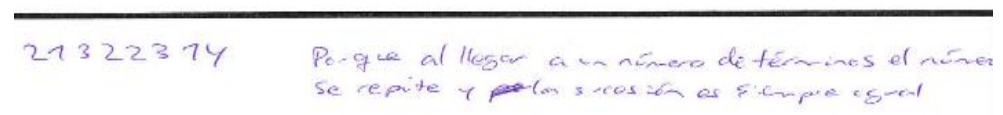

Figura 6. Justificación de un grupo al tercer apartado de Tarea 1.1

Los alumnos están generando la idea de que la sucesión es periódica, por lo que el razonamiento que se está poniendo en juego es abductivo. Basta representar el modelo de Toulmin asociado a las respuestas para ver que en este caso se trata, en particular, de una abducción por patrones (Figura 7).

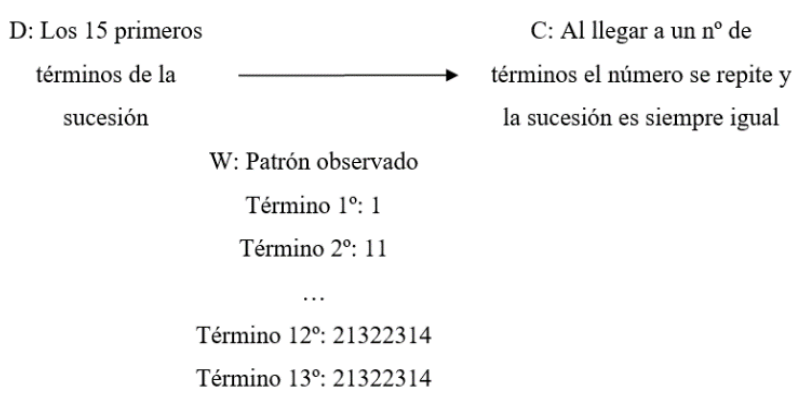

Figura 7. Modelo de Toulmin de una respuesta a Tarea 1.1

Tarea 1.2. De nuevo, todos los grupos calculan correctamente los términos solicitados y justifican de forma parecida a la de la tarea anterior (los dos grupos que no aportan justificaciones siguen sin hacerlo). En esta tarea cada grupo parte de un valor inicial diferente y en todos los casos la sucesión se estabiliza en un solo valor, aunque el momento a partir del que esto sucede varía. Los grupos se expresan de forma prácticamente idéntica a la tarea anterior. De hecho, uno de ellos hace mención explícita a que es la "misma sucesión" (Figura 8) y otro utiliza la expresión "entrar en bucle" para referirse a la periodicidad.

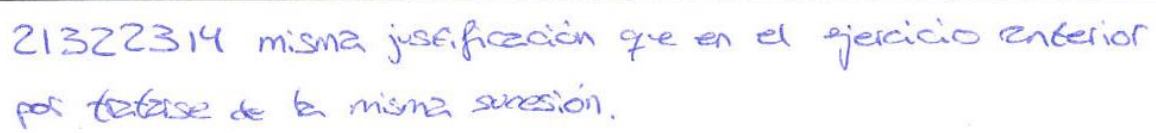

Figura 8. Justificación del grupo 1 al tercer apartado de Tarea 1.2

Los grupos razonan de modo muy similar a la tarea previa, por lo que aparecen mayoritariamente abducciones por patrones. Sin embargo, destaca la diferencia de justificación del grupo 1, que en este caso es una abducción por analogía (Figura 9).

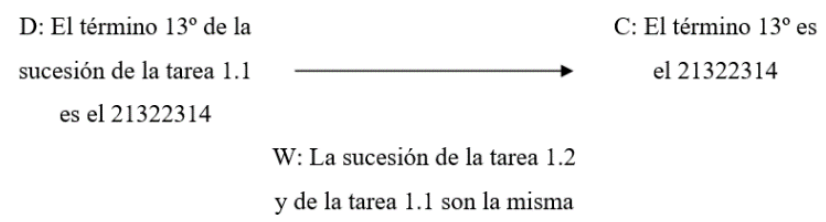

Figura 9. Modelo de Toulmin de una respuesta a Tarea 1.2 
Tarea 1.3. Aparecen errores en el cálculo de los términos de la sucesión. Dos grupos calculan mal el segundo término cometiendo el mismo error, que consiste en ordenar mal. Por ejemplo, un grupo escribe 111715 en lugar de 111517. El único grupo que calcula mal el término $13^{\circ}$ no es ninguno de los anteriores. El error en este caso consiste en omitir parte del término, escribiendo 611213141718 cuando debería ser 6122132425161718. Dado que estos errores aparecen solo de manera puntual, pueden ser achacables a despistes en el proceso de transcripción y de recuento.

En el tercer apartado de la tarea todos los grupos calculan correctamente el término $100 .^{\circ}$ (teniendo en cuenta que el grupo que calculó mal el $13 .^{\circ}$ es coherente con su error). Dos grupos siguen sin aportar ningún tipo de justificación o explicación.

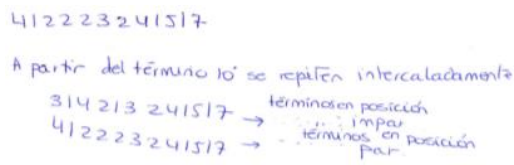

Figura 10. Respuesta de grupo 7 al tercer apartado de Tarea 1.3

Tabla 2. Transcripciones literales de justificaciones de grupos en el $3 .{ }^{\text {er }}$ apartado de Tarea 1.3

\begin{tabular}{cl}
\hline Grupo & \multicolumn{1}{c}{ Justificación } \\
\hline 1 & A partir del 14 los pares llevan este número y los impares... \\
2 & La sucesión sigue repitiéndose en el mismo número. En este caso al ser par son $2-2$ \\
3 & Porque todos se repiten a partir del $n .^{\circ} 6$ \\
4 & A partir del $10 .^{\circ}$ los pares son así y los impares son como el $13 .^{\circ}$ \\
5 & [En blanco] \\
6 & Se repiten dos números, uno para los pares y otro para los impares \\
7 & A partir del término 10 se repiten intercaladamente \\
8 & $\begin{array}{l}\text { A partir del número } 13 \text { los números pares llevan esta sucesión y los impares la ante- } \\
\text { rior }\end{array}$ \\
& [En blanco] \\
10 & $\begin{array}{l}\text { Cada dos números se repite el mismo número, uno corresponde a los pares y el otro } \\
\text { a los impares }\end{array}$
\end{tabular}

Al ser la situación un poco más compleja, las explicaciones comienzan a ser más diversas (Tabla 2). Tres de los grupos no mencionan explícitamente el momento a partir del cual la sucesión se "estabiliza". El grupo 2 tiene problemas para explicarse pues dice que sigue repitiéndose el mismo número, pero luego habla del caso par. Todos los grupos hacen mención explícita a términos pares e impares, sólo el grupo 7 (Figura 10) utiliza una expresión genérica: "repetirse intercaladamente". En esta tarea, en todas las respuestas satisfactorias aparecen razonamientos de tipo abductivo, más concretamente encontramos abducciones por patrones (Figura 11). 


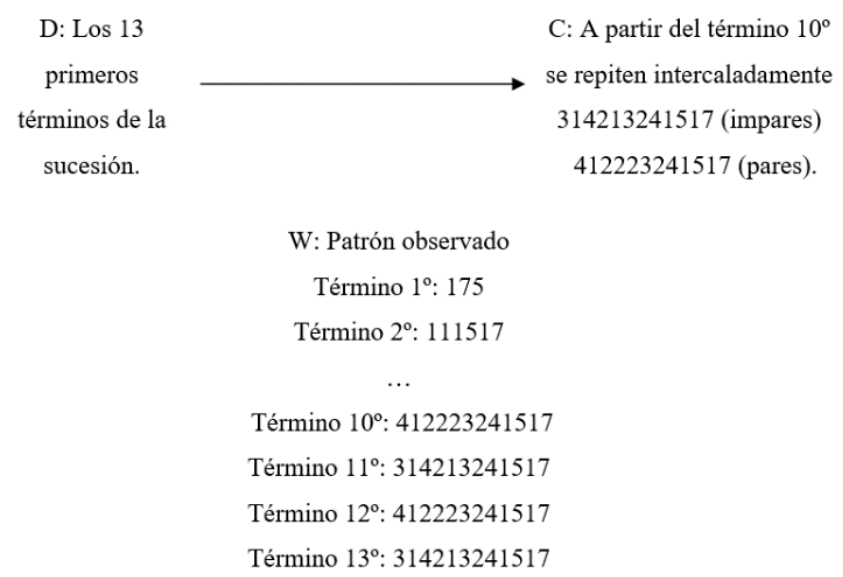

Figura 11. Modelo de Toulmin de la respuesta de grupo 7 a Tarea 1.3

\subsection{Resultados de la Tarea 2}

Tarea 2.1. Puesto que a partir de esta tarea los grupos ya disponían de la herramienta informática para realizar los cálculos, desaparecen errores de transcripción y recuento. Esta tarea implicaba la selección libre, por parte de los alumnos, de los valores iniciales de las sucesiones estudiadas. En la Tabla 3 se dan los valores elegidos por cada uno de los 10 grupos. El grupo 9 sólo escribe un número y no rellena la ficha.

\begin{tabular}{cc} 
Tabla 3. Valores iniciales elegidos por cada grupo en Tarea \\
\cline { 2 - 2 } Grupo & Valores \\
\hline 1 & $12,7,10,5,4,6$ \\
2 & $21,100,70,29,84,22$ \\
3 & $17,211,1408,99,63882,777$ \\
4 & $761,11121314,581,12486,879,91234$ \\
5 & $28,224,99,19,712,100$ \\
6 & $47,73,69,83,42,22$ \\
7 & $1711,0211,0810,2002,45,2018$ \\
8 & $22,333,10,123,24,555$ \\
9 & 555 \\
10 & $465,15,5730,999,333,0$ \\
\hline
\end{tabular}

Los alumnos propusieron mayoritariamente números de 2 y de 3 cifras (Figura 12). Resulta interesante la aparición muy numerosa de la cifra 0 (de los 55 números propuestos, 10 incluyen 0 ). También es reseñable que 3 grupos han abordado el caso del 22 que es el único valor para el que la sucesión es constante. 


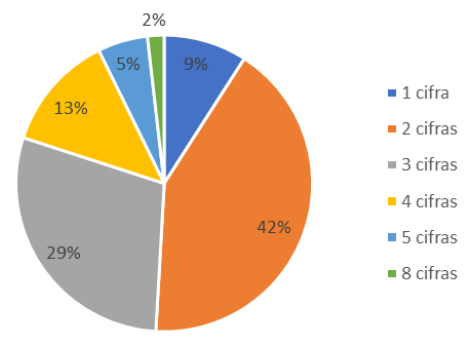

Figura 12. Distribución del número de cifras en los valores iniciales elegidos en Tarea 2.1

Tarea 2.2. Tenía un carácter totalmente abierto y en ella se pretendía recoger las conclusiones a las que los alumnos pudieran haber llegado tras su trabajo anterior. En la Tabla 4 se transcriben las conjeturas de cada uno de los grupos.

Tabla 4. Trascripciones literales de las conjeturas formuladas por los grupos en Tarea 2.2

\begin{tabular}{|c|c|}
\hline Grupo & Justificación \\
\hline 1 & $\begin{array}{l}\text { Al cabo de varios números, la sucesión comienza un módulo que se repite cada } x \\
\text { números }\end{array}$ \\
\hline 2 & Los números que forman el propio término seguro que aparecen en la sucesión \\
\hline 3 & Llega algún punto en el que dejan de ser todos diferentes y pasan a seguir un patrón \\
\hline 4 & Todos ellos, al llegar a cierto número, convergen a un valor cíclico de números \\
\hline 5 & Llegados a un número los términos comienzan a repetirse o a alternarse \\
\hline 6 & Son convergentes y repiten y mantienen un término \\
\hline 7 & $\begin{array}{l}\text { Ciertas sucesiones llegadas a cierto término se repiten. Otras se repiten intercala- } \\
\text { damente (de } 1, \text { de } 2 \text { y de } 3 \text { ) }\end{array}$ \\
\hline 8 & A partir de cierto número se repite la sucesión \\
\hline 9 & El número varia hasta llegar $+o-$ al $a_{10}$ que se repite de forma periódica \\
\hline 10 & Entra en bucle a partir de un término característico de cada valor inicial \\
\hline
\end{tabular}

Todos los grupos menos uno (grupo 2) orientan su conjetura hacia el comportamiento asintótico. Dos grupos (6 y 8) conjeturan que la sucesión es eventualmente constante y siete grupos (1, 3, 4, 5, 7, 9 y 10) conjeturan que se llega a ciclos. De ellos, sólo dos (5 y 7) concretan las posibles longitudes de los ciclos. El grupo 9 (Figura 13) hace una estimación del tiempo que tardan en aparecer los ciclos.

$$
\begin{aligned}
& \overline{\text { El nuimero raria hasta llegar to- al }} \\
& a_{10} \text { que se repite de forma periódica }
\end{aligned}
$$

Figura 13. Respuesta del grupo 9 a Tarea 2.2

Si bien el razonamiento abductivo es el que se pone en juego a la hora de elaborar una conjetura, esta ha sido obtenida en las tres primeras tareas, por lo que se considera que el razonamiento abductivo no predomina en esta actividad. Las Tareas 2.1 y 2.2 tienen como objetivo el someter dicha conjetura a pruebas mediante la calculadora look and say. Por ello, los razonamientos en uso son inductivos (Figura 14). 


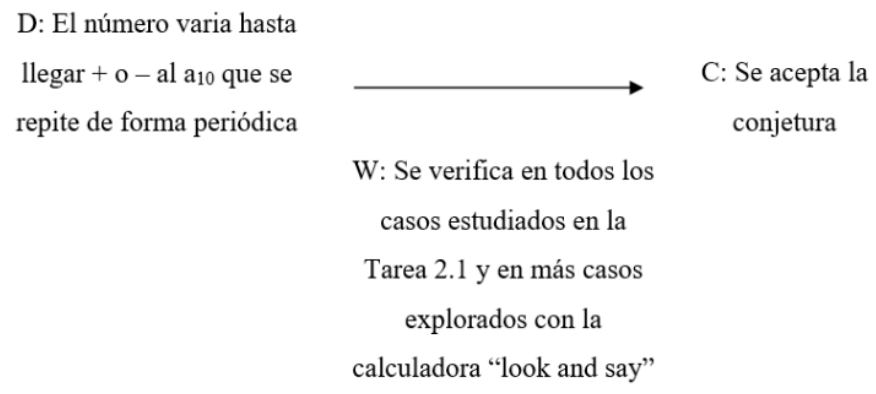

Figura 14. Modelo de Toulmin de la respuesta del grupo 9 a Tarea 2.2

\subsection{Resultados de la Tarea 3}

Tarea 3.1. Aunque al igual que en la Tarea 2.2 estamos ante una situación abierta, aquí la variedad de respuestas es mayor debido a que se trata de la primera tarea de esta segunda actividad. La Tabla 5 recogen los enunciados dados por cada grupo.

Tabla 5. Transcripción literal de las características señaladas por los grupos en Tarea 3.1

\begin{tabular}{|c|c|}
\hline Grupo & Características \\
\hline 1 & [En blanco] \\
\hline 2 & [En blanco] \\
\hline 3 & $\begin{array}{l}\text { Cada sucesión sigue distinto patrón, ya sea de dos en dos, de tres en tres, ... de una } \\
\text { en una... } \\
\text { Todas acaban en el mismo número } \\
\text { Todas tienen un número par de cifras }\end{array}$ \\
\hline 4 & $\begin{array}{l}\text { Acaban en el número más grande que tienen } \\
\text { Todos, salvo el primero, tienen un número par de dígitos }\end{array}$ \\
\hline 5 & Todos tienen un número par de cifras \\
\hline 6 & $\begin{array}{l}\text { Tienen siempre un número par de cifras, van en parejas } \\
\text { Las parejas se colocan en orden respecto al segundo miembro }\end{array}$ \\
\hline 7 & $\begin{array}{l}\text { Todos ellos son números naturales } \\
\text { El número de cifras con respecto a los términos varía de dos en dos cada dos térmi- } \\
\text { nos } \\
\text { El número de cifras es siempre par y como mucho pueden ser } 20\end{array}$ \\
\hline 8 & $\begin{array}{l}\text { A partir de cierto número se repite la sucesión } \\
\text { El número de cifras salvo el primero en algunos casos es siempre par } \\
\text { En cada número las cifras pares van en orden creciente }\end{array}$ \\
\hline 9 & Que al final se sigue repitiendo constantemente el mismo número o varios \\
\hline 10 & $\begin{array}{l}\text { El número o números que se empiezan a repetir tienen } 20 \text { dígitos o menos, puesto } \\
\text { que hay un límite combinaciones posibles con } 20 \text { dígitos }\end{array}$ \\
\hline
\end{tabular}

El grupo 9 no habla de características de los elementos, sino que solo vuelve sobre la "periodicidad". Los grupos 3 y 8 repiten este tema, pero también aportan otras propiedades referidas a los elementos. Así pues, son siete los grupos que señalan, al menos, una característica o propiedad sobre los elementos de la sucesión. De ellos, dos (el 5 y el 10) únicamente señalan una característica, otros cuatro (el 3, el 4, el 6 y el 8) señalan dos características y uno (el 7) señala cuatro características. 


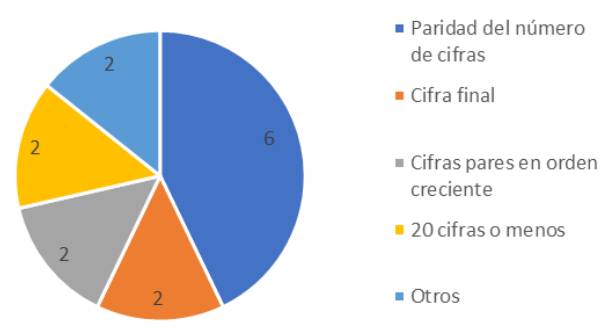

Figura 15. Características señaladas por los grupos en Tarea 3.1

Como vemos en la Figura 15, la propiedad más repetida es la paridad del número de cifras. De los seis grupos que lo mencionan, dos concretan que es así salvo quizás para el primer término. Los dos enunciados clasificados como "otros" provienen del grupo 7. En la Figura 16 vemos las características dadas por uno de los grupos.

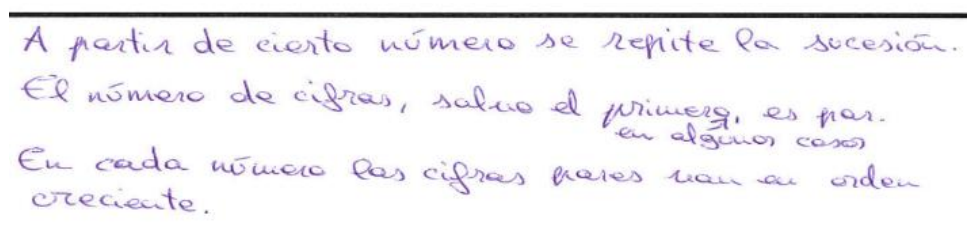

Figura 16. Respuesta del grupo 8 a Tarea 3.1

Tarea 3.2. En el primer apartado, había una solución única no trivial y las respuestas de todos los grupos fueron correctas. En el segundo apartado, había múltiples soluciones no triviales, lo que conlleva mayor variedad en las respuestas de los alumnos. Un grupo lo deja en blanco. Todos los demás logran dar una solución, si bien sólo cuatro de ellos dan todas las opciones posibles (permutaciones de una solución cualquiera, ver Figura 17). El grupo 8 escribe que "según el orden de los dígitos tiene distintas soluciones".

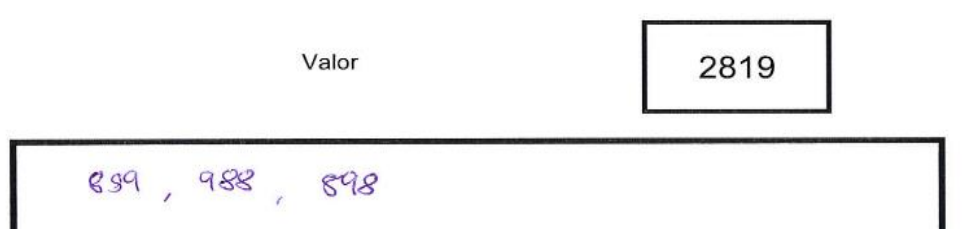

Figura 17. Todos los posibles anteriores al 2819 en Tarea 3.2

En el tercer apartado no existía un término anterior no trivial al valor dado, aunque por razones distintas según los grupos. En los grupos 1, 2, 3, 6, 8 y 10 se debía al orden de las cifras, y en los grupos 4, 5, 7 y 9 a la cantidad de cifras. La mayor dificultad se ve reflejada en que tres grupos la dejan en blanco. Otros dos grupos dan un posible término anterior que no tiene sentido. Dos grupos (en los que el único problema está en el orden) dan posibles anteriores que son correctos si la sucesión no tuviera en cuenta el orden. Un grupo da una solución trivial correcta (Figura 18). 


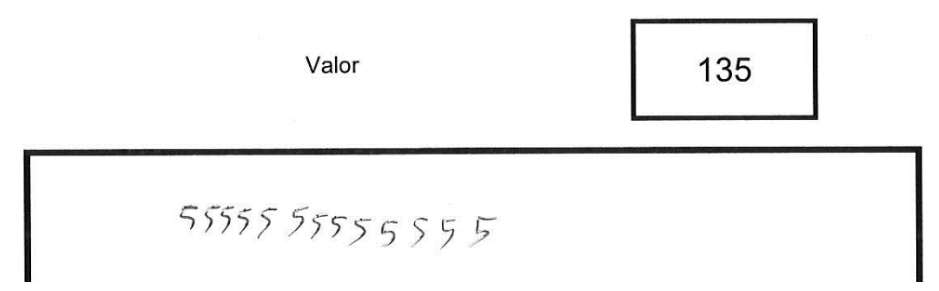

Figura 18. Término anterior trivial en Tarea 3.2

Tienen especial interés las respuestas de los alumnos que podían vislumbrar que no existía elemento anterior no trivial. Sólo dos grupos señalan que no hay término anterior, aunque ninguno aporta justificación explícita en sus producciones escritas. No obstante, en la producción de uno de ellos, el grupo 3, sí se puede interpretar algún tipo de uso implícito de razonamiento de tipo deductivo para concluir que el término anterior no existe utilizando las propiedades descritas en la Tarea 3.1 (Figura 19).

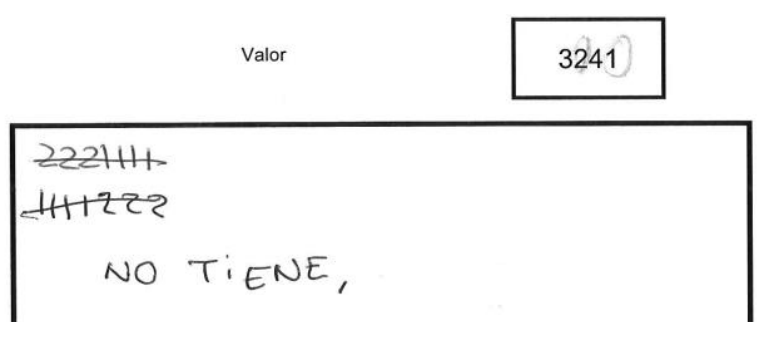

Figura 19. Respuesta de grupo 3 a Tarea 3.2

El argumento utilizado para determinar que no existe término anterior no es explícito, pero se puede deducir de las marcas realizadas en el valor dado (Figura 20).

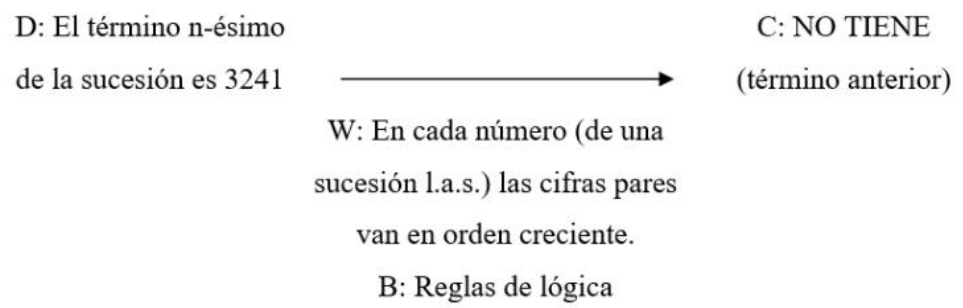

\section{C: NO TIENE}

(término anterior)

Figura 20. Modelo de Toulmin de la respuesta de grupo 3 a Tarea 3.2

Tarea 3.3. En el primer apartado hay al menos una solución no trivial. Dos grupos no lo abordan correctamente: uno lo deja en blanco y el otro sólo da el término anterior (y no el anterior del anterior). De los ocho grupos restantes, la mayoría (seis) se limitan a dar una posibilidad correcta para cada término pedido. Únicamente dos grupos dan todas las posibilidades correctas no triviales para los dos valores (Figura 21).

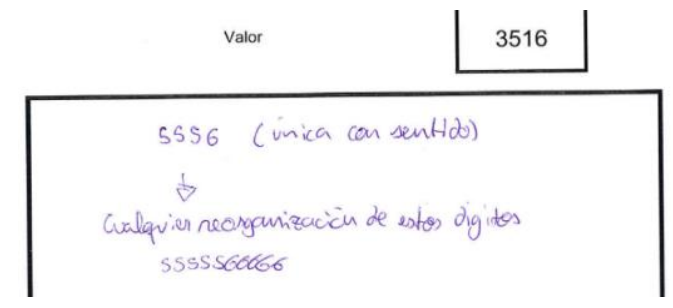

Figura 21. Respuesta correcta al primer apartado de Tarea 3.3 
En el segundo apartado no hay solución no trivial para el término pedido, aunque en todos los casos existe al menos un término anterior no trivial al dado. Un grupo sigue dejando en blanco el apartado, mientras que otro no da correctamente el valor anterior. De los ocho grupos que dan correctamente un término anterior al dado, solo cuatro escriben algo sobre el término "anterior del anterior". Dos grupos dan una solución trivial (Figura 22) y los otros dos grupos afirman sin justificación explícita que no existe el término anterior al anterior.

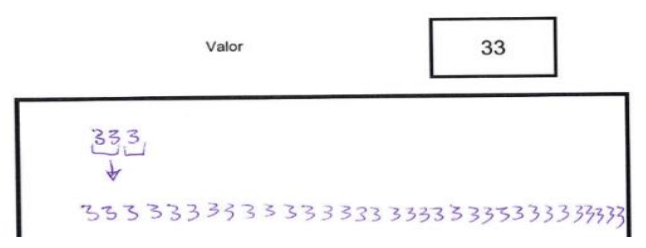

Figura 22. Respuesta trivial correcta al segundo apartado de Tarea 3.3

Como en la Tarea 3.2, nos centramos en la respuesta del grupo 3. Este grupo ha dado un valor incorrecto del término anterior; sin embargo, en su respuesta se intuye la utilización de un razonamiento de tipo deductivo que les permite concluir que no existe término anterior del anterior (Figura 23).

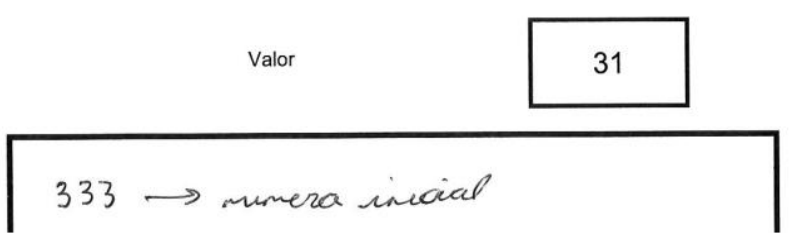

Figura 23. Respuesta de grupo 3 a Tarea 3.3

En la Tarea 3.1. este grupo señaló (Tabla 5) que todos los términos de la sucesión deben tener un número par de cifras. En su respuesta, parecen hacer uso de esta propiedad al indicar que el término 333 debe ser el "número inicial" y por tanto no tiene anterior (Figura 24).

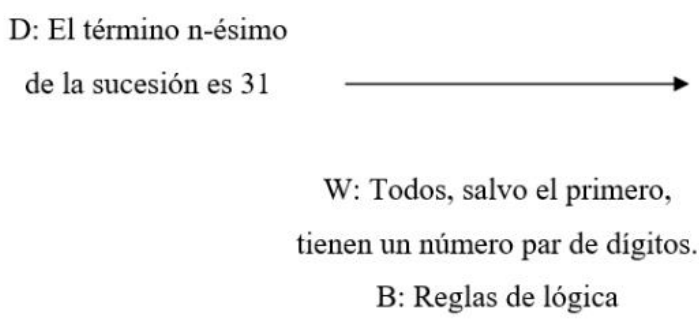

W: Todos, salvo el primero, tienen un número par de dígitos. B: Reglas de lógica

Figura 24. Modelo de Toulmin de la respuesta de grupo 3 a Tarea 3.3

\section{Discusión}

El trabajo con tareas basadas en las sucesiones look and say permite abordar diversos elementos del currículo de matemáticas de secundaria, sirven como contraejemplos de muchas concepciones erróneas detectadas en estudiantes vinculadas al concepto de sucesión (Przenioslo, 2006) y, además, coincidimos con Barton et al. (2004) en que no requieren de conocimientos específicos y se plantean fácilmente. 
La descomposición genética del concepto de sucesión en Bajo Benito et al. (2019) ha resultado útil en el diseño y secuenciación de las actividades. En este sentido, este trabajo proporciona una cierta validación experimental de la misma (Asiala et al., 1996) incluso en el contexto de las sucesiones look and say, que no coincide exactamente con el considerado por los autores anteriores. Por ejemplo, hemos visto que nuestro diseño no tenía en consideración distintos modos de representación o que ha sido necesario ampliar los prerrequisitos. Por ello, pensamos que la consideración de sucesiones similares a la utilizada en este trabajo puede servir para completar la descomposición genética del concepto de sucesión como lista numérica. Podría ser interesante mejorar el diseño de nuestras tareas incorporando diferentes modos de representación (Weigand, 2004) que permitan a los estudiantes establecer razonamientos visuales (Alcock y Simpson, 2004).

Bajo Benito et al. (2019) indican que las sucesiones recurrentes son un elemento matemático clave ligado al concepto de sucesión como lista numérica y se vinculan a niveles más avanzados de comprensión del concepto de sucesión que el trabajo con términos generales. Por otro lado, la realización de tareas basadas en estas sucesiones puede contribuir a reducir la identificación errónea entre los conceptos de sucesión numérica y progresión señalada, por ejemplo, en Bajo Benito et al. (2015).

Las sucesiones look and say han supuesto un contexto adecuado para el diseño de actividades de alta demanda cognitiva en las que intervienen los tres tipos de razonamiento de Pierce, especialmente el abductivo y el inductivo. En cuanto al razonamiento deductivo, está presente en las tareas asociadas al estudio del problema inverso, que han podido ser abordadas exitosamente por algunos alumnos. Otro tipo de tareas con razonamiento deductivo estarían vinculadas a la demostración de las conjeturas generadas en la Tarea 2. Por su naturaleza, este tipo de tarea excede el nivel de secundaria, si bien se han llevado a cabo a nivel universitario (Barton et al., 2004).

La implementación de la secuencia transcurrió sin incidentes y los investigadores que intervinieron en su desarrollo observaron una alta implicación de los alumnos, que se incrementó en las tareas en que se utilizaron medios tecnológicos, en la línea de lo reportado en el trabajo de Weigand (2004). Por otro lado, constataron dificultades en algunos de los estudiantes a la hora de verbalizar sus conjeturas y justificaciones.

En las Tareas 1 y 2, los alumnos fueron capaces de abordar con éxito las tareas de carácter más mecánico o algorítmico, relacionadas con el cálculo de términos de la sucesión. La estructuración de la secuencia de actividades en distintas tareas se reveló adecuada; prácticamente la totalidad de los grupos terminaron por formular personalmente la conjetura y aceptar la propiedad de periodicidad de las sucesiones.

En la Tarea 3.1, en la que se pide indicar propiedades o características especiales de los elementos de las sucesiones más allá de la propiedad de periodicidad, se ha encontrado una relativa riqueza en las respuestas de los alumnos (Tabla 4), incluso señalando propiedades no esperadas por los investigadores al diseñar la actividad. En este sentido, es destacable el hecho de que muchas de las conjeturas realizadas van en la línea de identificar patrones o características "estructurales" de los términos.

Por otra parte, consideramos que especialmente la Tarea 3 constituye una actividad de alta demanda cognitiva. Entre otras cosas requiere de los alumnos pensamiento no algorítmico, así como que exploren y comprendan la naturaleza de los conceptos matemáticos, procesos o relaciones (Smith y Stein, 1998). 
Las respuestas de algunos alumnos (especialmente los del grupo 10) muestran rasgos interesantes. Por ejemplo, en el segundo apartado de la Tarea 3.2 (Figura 17) dan todos los resultados correctos posibles, mientras que en el primer apartado de la Tarea 3.3 (Figura 21) hacer esto llevaría mucho tiempo por lo que cambian de estrategia dando una solución e indicando que cualquier reorganización de esos dígitos también constituiría una solución válida. Del mismo modo en el segundo apartado de la Tarea 3.3 (Figura 22), al no poderse encontrar una solución al problema utilizando las técnicas que anteriormente han tenido éxito, se presenta una solución original.

Algunos de estos rasgos se corresponden con los señalados por Jaime y Gutiérrez (2017) en relación con alumnos de altas capacidades. Esto abre la puerta a estudiar la posibilidad de que esta secuencia de tareas pueda servir como punto de partida para el diseño de una herramienta de detección de altas capacidades en alumnos participantes.

\section{Agradecimientos}

A los revisores y las editoras, que han contribuido a mejorar sustancialmente el manuscrito. Al grupo Investigación en Educación Matemática S60_20R, Gobierno de Aragón.

\section{Referencias}

Aguayo, P. (2011). La teoría de la abducción de Peirce: Lógica, metodología e instinto. Ideas y valores, 60(145), 33-53.

Alcock, L. y Simpson, A. (2004). Convergence of sequences and series: Interactions between visual reasoning and the learner's beliefs about their own role. Educational Studies in Mathematics, 57, 1-32. https://doi.org/10.1023/B:EDUC.0000047051.07646.92

Arce, M. y Conejo, L. (2019). Razonamientos y esquemas de prueba evidenciados por estudiantes para maestro: Relaciones con el conocimiento matemático. En J. M. Marbán, M. Arce, A. Maroto, J. M. Muñoz-Escolano y Á. Alsina (Eds.), Investigación en Educación Matemática XXIII (pp. 163-172). SEIEM.

Arnal-Palacián, M. (2019). Límite infinito de una sucesión: fenómenos que organiza. Trabajo de Tesis Doctoral. Universidad Complutense de Madrid.

Arnon, I., Cottrill, J., Dubinsky, E., Oktaç, A., Roa Fuentes, S., Trigueros, M. y Weller, K. (2014). APOS theory. A framework for research and curriculum development in mathematics education. Springer. https://doi.org/10.1007/978-1-4614-7966-6

Asiala, M. B., DeVries, A., Dubinsky, D., Mathews, E. y Thomas, D. K. (1996). A framework for research and curriculum development in undergraduate mathematics education. En J. Kaput, A. H. Schoenfeld y E. Dubinsky (Eds.), Research in Collegiate Mathematics Education II (pp. 1-32). AMS. https://doi.org/10.1090/cbmath/006/01

Bajo Benito, J. M., Gavilán-Izquierdo, J. M. y Sánchez-Matamoros, G. (2019). Caracterización del esquema de sucesión numérica en estudiantes de Educación Secundaria Obligatoria. Enseñanza de las Ciencias, 37(3), 149-167.

Bajo Benito, J. M., Sánchez-Matamoros, G. y Gavilán Izquierdo, J. M. (2015). Las progresiones como indicador de la comprensión del concepto de sucesión numérica en alumnos de segundo ciclo de enseñanza secundaria obligatoria. En C. Fer- 
nández, M. Molina y N. Planas (Eds.), Investigación en Educación Matemática XIX (pp. 143-151). SEIEM.

Barton, J., Feil, D., Lartigue, D. y Mullins, B. (2004). Sequences for Student Investigation. PRIMUS, 14(4), 354-368. https://doi.org/10.1080/10511970408984099

Biza, I., Hewitt, D., Watson, A. y Mason, J. (2020). Generalization strategies in finding the $n$th term rule for simple quadratic sequences. International Journal of Science and Mathematics Education, 18, 1105-1126. https://doi.org/10.1007/s10763019-10009-0

Bronstein, V. y Fraenkel, A. S. (1994). On a curious property of counting sequences. American Mathematical Monthly, 101(6), 560-563. https://doi.org/10.1080/00029890.1994.11996991

Chace, A. B. (1986). The Rhind Mathematical Papyrus. NCTM.

Conway, J. H. (1986). The weird and wonderful chemistry of audioactive decay. Eure$k a, 46,5-18$.

Creswell, J. W. (2012). Educational research: Planning, conducting, and evaluating quantitative and qualitative research. Pearson.

De la Cueva, F. (2016). Taller de Talento Matemático. Entorno Abierto, 12, 5-7.

Delgado, M. L., Codes, M., Monterrubio, M. C. y González-Astudillo, M. T. (2014). El concepto de serie numérica. Un estudio a través del modelo de Pirie y Kieren centrado en el mecanismo "folding back". Avances de Investigación en Educación Matemática, 6, 25-44.

Dubinsky, E. y McDonald, M. A. (2001). APOS: A constructivist theory of learning in undergraduate mathematics education research. En D. Holton (Ed.), The teaching and learning of mathematics at university level (pp. 275-282). Springer. https://doi.org/10.1007/0-306-47231-7_25

Fernández-Plaza, J. A. y Simpson, A. (2016). Three concepts or one? Students' understanding of basic limit concepts. Educational Studies in Mathematics, 93, 315-332. https://doi.org/10.1007/s10649-016-9707-6

Gairín, J. M., Manero, V., Muñoz-Escolano, J. M., y Oller-Marcén, A. M. (2018). La sucesión look and say. VIII Congreso Iberoamericano de Educación Matemática. Libro de Actas, CB-603 (pp. 16-24). FESPM.

Jaime, A. y Gutiérrez, Á. (2017). Investigación sobre estudiantes con alta capacidad. En J. M. Muñoz-Escolano, A. Arnal-Bailera, P. Beltrán-Pellicer, M. L. Callejo y J. Carrillo (Eds.), Investigación en Educación Matemática XXI (pp.71-89). SEIEM.

Jojo, Z. M. M. (2014). Instructional design in the formation of mental images and the genetic decomposition of a concept. Journal of Educational and Social Research, 4(3), 139-148.

Manrique, V. H. y Soler-Álvarez, M. N. (2014) El proceso de descubrimiento en la clase de matemáticas: Los razonamientos abductivo, inductivo y deductivo. Enseñanza de las Ciencias, 32(2), 191-219. https://doi.org/10.5565/rev/ensciencias.1026

Peirce, C. S. (1934). The collected papers of Charles Sanders Peirce, Vol. V: Pragmatism and pragmaticism. C. Hartshorne \& P. Weiss (Eds.). Harvard University Press. 
Pedemonte, B. (2007). How can the relationship between argumentation and proof be analysed? Educational Studies in Mathematics, 66, 23-41. https://doi.org/10.1007/s10649-006-9057-X

Pedemonte, B. y Reid, D. (2011). The role of abduction in proving processes. Educational Studies in Mathematics, 76, 281-303. https://doi.org/10.1007/s10649-010-9275-0

Postic, M. y de Ketele, J. M. (1988). Observer les situations éducatives. Presses Universitaires de France.

Przenioslo, M. (2006). Conceptions of a sequence formed in secondary schools. International Journal of Mathematical Education in Science and Technology, 37(7), 805-823. https://doi.org/10.1080/00207390600733832

Saurberg, J. y Shu, L. (1997). The long and the short on counting sequences. The American Mathematical Monthly, 104(4), 306-317. https://doi.org/10.1080/00029890.1997.11990642

Sigler, L. E. (2002). Fibonacci's Liber Abaci. A translation into modern English of Leonardo Pisano's Book of Calculation. Springer. https://doi.org/10.1007/978-1-4613-0079-3

Smith, M. S. y Stein, M. K. (1998). Selecting and creating mathematical tasks: From research to practice. Mathematics Teaching in the Middle School, 3(5), 344-350. https://doi.org/10.5951/MTMS.3.5.0344

Toulmin, S. E. (2003). The uses of argument. Updated edition. Cambridge University Press. https://doi.org/10.1017/CBO9780511840005

Trigueros, M. y Oktaç, A. (2019). Task design in APOS theory. Avances de Investigación en Educación Matemática, 15, 43-55. https://doi.org/10.35763/aiem.v0i15.256

Weigand, H. G. (2004). Sequences - basic elements for discrete mathematics. ZDM, 36(3), 91-97. https://doi.org/10.1007/BF02652776

Yeo, J. (2007). Mathematical tasks: Clarification, classification and choice of suitable tasks for different types of learning and assessment. Technical Report ME2007-01. NIE Singapore.

Yin, R. K. (2018). Case Study research and applications. Sage.

\section{Referencias de los autores}

Víctor Manero, Universidad de Zaragoza (España). vmanero@unizar.es

José M. Muñoz-Escolano, Universidad de Zaragoza (España). jmescola@unizar.es

Antonio M. Oller-Marcén, Centro Universitario de la Defensa de Zaragoza (España).

oller@unizar.es 


\title{
Design and implementation of cognitive high-demand tasks based on the look and say sequence
}

\author{
Víctor Manero, Universidad de Zaragoza. \\ José M. Muñoz-Escolano, Universidad de Zaragoza. \\ Antonio M. Oller-Marcén, Centro Universitario de la Defensa de Zaragoza.
}

The school treatment of sequences is usually focused on computations and, in particular, centered in computations involving arithmetic and geometric progressions. This treatment produces a wrong identification between numerical sequences and progressions. Thus, it might be interesting to introduce in the classroom numerical sequences that can be easily defined, but which are neither arithmetic nor geometric progressions. For this purpose, we decide to use the so-called 'ordered look and say' sequences. These sequences are a version of the 'look and say' sequences introduced and popularized by Conway (1986). Our main goal was to design and implement a set of tasks dealing with these sequences. In the design process, we took into account the genetic decomposition of the concept of sequence and the cognitive demand levels of the tasks. In addition, we wanted the activities to promote the three types of reasoning defined by Peirce (deductive, inductive and abductive). The tasks were implemented in a 90 minutes session with 10th graders (15-16 years) enrolled in the "Taller de Talento Matemático" program, which takes place at the University of Zaragoza and involves extracurricular lectures and workshops carried out with students with great interest in mathematics. The written productions of these students were later analyzed. We observed the three types of reasoning in the student productions. Most students were able to complete tasks with a high level of cognitive demand, even though some showed difficulties verbalizing their conjectures and justifications. The theoretical tools used in order to design the tasks (genetic decomposition, cognitive demand levels) revealed useful in the process. The structure and sequencing of the tasks were also helpful in order to help the students in completing all the tasks and in stating their own conjectures. Toulmin diagrams were used to analyze some aspects of student responses. Finally, we observe that some responses include features common in students with high mathematical skills (Jaime y Gutiérrez, 2017). Hence, the designed tasks could eventually serve to identify mathematically gifted students. 